\title{
Archimedean tiling graphs with Gallai's property
}

\author{
Zhikui Chang and Liping Yuan
}

\begin{abstract}
Gallai in 1966 raised the question about the existence of graphs with the property that every vertex is missed by some longest path. This property will be called Gallai's property. In this paper we present some graphs embeddable into Archimedean tiling graphs, with both connectivity 1 and 2, satisfying Gallai's property.
\end{abstract}

\section{Introduction}

In 1966 Gallai [2] raised the question whether (connected) graphs do exist such that each vertex is missed by some longest path. This property will be called Gallai's property.

In 1969, Walther [13] firstly constructed such a graph with 25 vertices, which has connectivity 1 . In 1975, Schmitz [10] found a planar graph with 17 vertices satisfying Gallai's property, which is the smallest planar graph with connectivity 1 up to now.

In 1972, Zamfirescu in [15] asked about examples with higher connectivity, and presented the first 2-connected planar graph with 82 vertices satisfying Gallai's property. Soon a smaller example with 32 vertices was given in [16]. In 1996, Skupień [11] found a 2-connected graph with 26 vertices satisfying Gallai's property, which is the smallest 2-connected graph so far.

Key Words: Gallai's property, Archimedean tiling graphs, the longest path.

2010 Mathematics Subject Classification: Primary 05C38; Secondary 05C62.

Received: April, 2016

Revised: April, 2016.

Accepted: April, 2016. 
There exist large classes of graphs without Gallai's property. Klavžar and Petkovšek [7] proved that split graphs and cacti are among them. In [8] it is shown that no grid graph has Gallai's property.

Motivated by Menke's negative result, Nadeem et al. [9] considered the family of all graphs embeddable in planar lattice or regular hexagonal lattice, and found that Gallai's question again receives a positive answer. And the embeddings in cubic lattice [1] and regular triangular lattice [6] have also been studied.

For a general overview of tilings, see Grünbaum and Shephard's book [5]. A plane tiling $\mathcal{T}$ is a countable family of closed sets $\mathcal{T}=\left\{T_{1}, T_{2}, \cdots\right\}$ which cover the plane without gaps or overlaps. And every closed set $T_{i} \in \mathcal{T}$ is called a tile of $\mathcal{T}$. We consider a special case of tilings in which each tile is a polygon. If the corners and sides of a polygon coincide with the vertices and edges of the tiling, we call the tiling edge-to-edge. A so-called type of vertex describes its neighbourhood. If, for example, in some cyclic order around a vertex there are a triangle, then another triangle, then a square, next a third triangle, and last another square, then its type is $\left(3^{2} .4 .3 .4\right)$. We consider plane edge-to-edge tilings in which all tiles are regular polygons, and all vertices are of the same type. Thus, the vertex type will be defining our tiling.

There exist precisely eleven such tilings [5]. These are $\left(3^{6}\right),\left(3^{4} .6\right),\left(3^{3} \cdot 4^{2}\right)$, $\left(3^{2} .4 .3 .4\right),(3.4 .6 .4),(3.6 .3 .6),\left(3.12^{2}\right),\left(4^{4}\right),(4.6 .12),\left(4.8^{2}\right)$, and $\left(6^{3}\right)$. They are called Archimedean tilings. The graph formed by an Archimedean tiling is called an Archimedean tiling graph. For the sake of convenience, we still use the notation for an Archimedean tiling, such as ( $\left.3^{4} .6\right)$, to denote the corresponding Archimedean tiling graph.

Clearly, the lattice graph, the regular triangular lattice graph and the regular hexagonal lattice graph are all Archimedean tiling graphs. As a result, we are motivated to consider the family of graphs embeddable in the other 8 Archimedean tiling graphs, and obtain positive answers to Gallai's question for both connectivity 1 and 2 .

\section{Embeddings of graphs with connectivity 1}

Let $G$ be a graph homeomorphic to the graph $G^{\prime}$ in Figure 1. For each edge of $G^{\prime}$ the corresponding path of $G$ has a number of vertices of degree 1 and 2, denoted by $x, y, z, t, w, m$ respectively, also see Figure 1 .

We need the following strengthening of Lemma 1 in [9], the proof of which is similar and therefore omitted.

Lemma 2.1. The longest paths of $G$ have empty intersection if $0 \leq m \leq$ $\min \{y, z\}, 2 x \geq y+2 z+1, t \geq y+2 z-m+1, t \geq x+z+1, t \geq y+m+1$, 


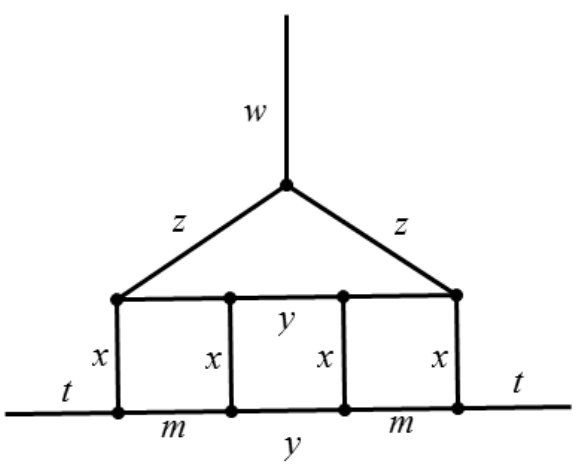

Figure 1: The graph $G^{\prime}$.

and $w=x+t-z$.

Theorem 2.2. There exists a subgraph of $\left(3^{4} .6\right)$ with 62 vertices satisfying Gallai's property.

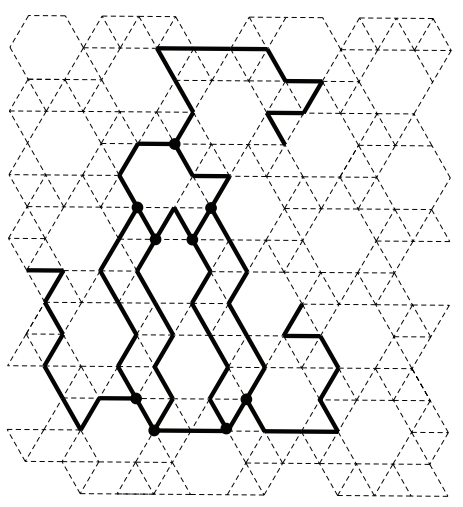

Figure 2: An embedding of $G$ in $\left(3^{4} \cdot 6\right)$.

Proof. If $x=5, t=8, z=2, w=11, y=1$ and $m=0$, then the conditions in Lemma 2.1 hold. Thus the corresponding graph $G$ of order 62 satisfying Gallai's property. Figure 2 shows an embedding of $G$ in $\left(3^{4} .6\right)$.

Theorem 2.3. The Archimedean tiling graph $\left(3^{3} .4^{2}\right)$ contains a subgraph of order 46 satisfying Gallai's property. 


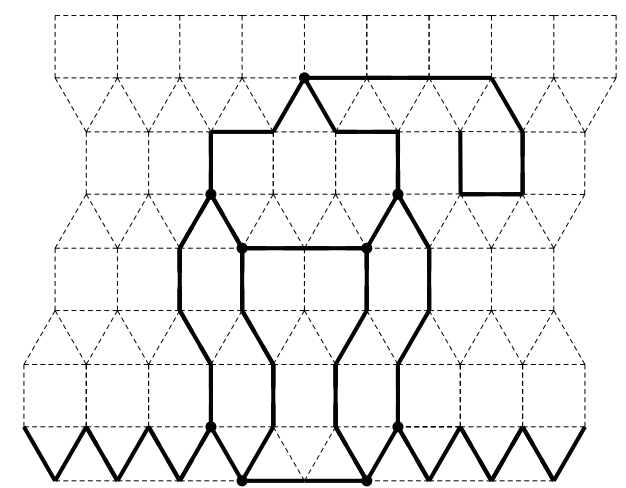

Figure 3: An embedding of $G$ in $\left(3^{3} \cdot 4^{2}\right)$.

Proof. It is easy to see that $G$ with $x=3, t=6, z=2, w=7, y=1$ and $m=0$ satisfies the conditions of Lemma 2.1. It has order 46 and is embeddable in $\left(3^{3} \cdot 4^{2}\right)$, as shown in Figure 3 .

Theorem 2.4. There is a graph of order 48 embedded in (32.4.3.4) satisfying Gallai's property.

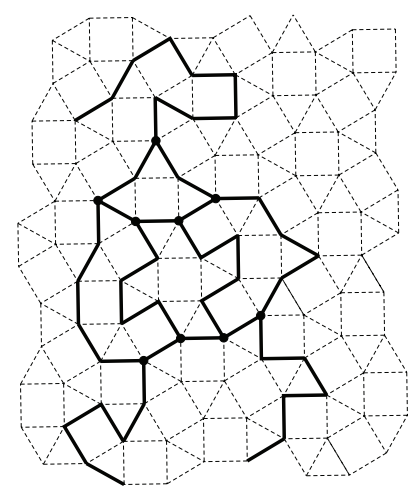

Figure 4: An embedding of $G$ in $\left(3^{2} .4 .3 .4\right)$.

Proof. In Lemma 2.1 we take $x=4, t=6, z=1, w=9, y=0$ and $m=0$, then all the conditions could be verified. Figure 4 exhibits an embedding of the corresponding $G$ with 48 vertices in $\left(3^{2} .4 .3 .4\right)$. 
Theorem 2.5. In (3.6.3.6) there is a subgraph of order 92 satisfying Gallai's property.

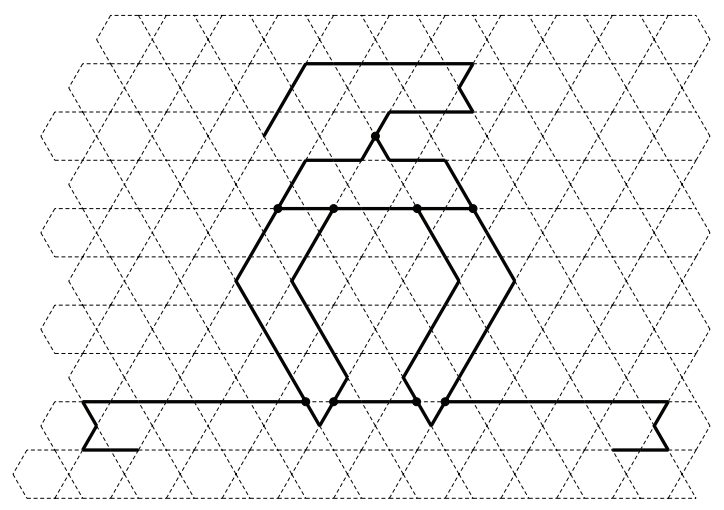

Figure 5: An embedding of $G$ in (3.6.3.6).

Proof. We consider Lemma 2.1 by setting $x=7, t=12, z=4, w=15, y=2$ and $m=1$. Clearly all the conditions are satisfied. Then we obtain a graph $G$ of order 92, and one of its embedding in (3.6.3.6) is shown in Figure 5.

Theorem 2.6. There exists a subgraph of (3.4.6.4) of order 100 satisfying Gallai's property.

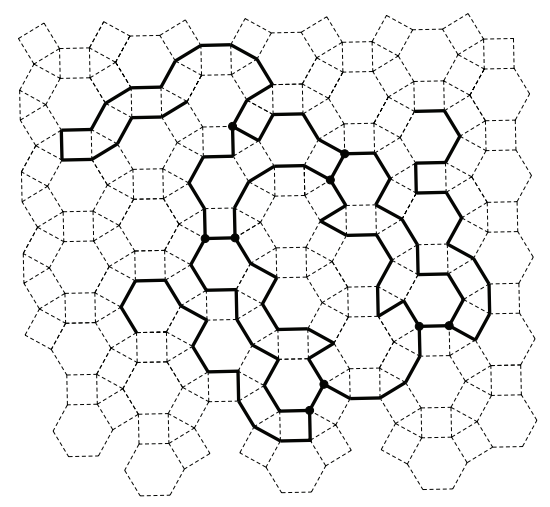

Figure 6: An embedding of $G$ in (3.4.6.4). 
Proof. In Lemma 2.1 we take $x=8, t=13, z=4, w=17, y=4$ and $m=0$, then we get a graph $G$ of order 100 having Gallai's property. Figure 6 reveals an embedding of $G$ in (3.4.6.4) tiling graph.

Theorem 2.7. In $\left(4.8^{2}\right)$ there is a subgraph of order 166 satisfying Gallai's property.

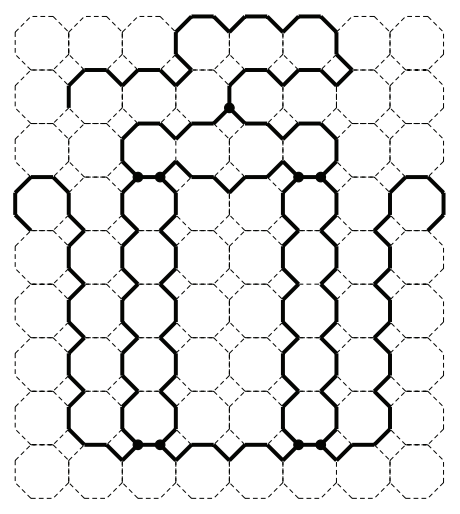

Figure 7: An embedding of $G$ in $\left(4.8^{2}\right)$.

Proof. If $x=14, t=22, z=7, w=29, y=7$ and $m=0$, then the conditions in Lemma 2.1 are verified. Thus we get a graph $G$ of order 166, and the embedding of it in the tiling graph $\left(4.8^{2}\right)$ could be seen in Figure 7 .

Theorem 2.8. The Archimedean graph (4.6.12) has a subgraph of order 207 satisfying Gallai's property.

Proof. Again consider a particular case of Lemma 2.1. Set $x=15, t=30$, $z=11, w=34, y=7$ and $m=2$. Then it is easy to check the conditions in Lemma 2.1 are satisfied. Hence we have a graph $G$ of order 207 satisfying Gallai's property, and an embedding of $G$ is shown in Figure 8.

Theorem 2.9. There is a subgraph of order 191 embedded in $\left(3.12^{2}\right)$ satisfying Gallai's property.

Proof. It is clear that $G$ with $x=15, t=25, z=8, w=32, y=8$ and $m=2$ satisfies the conditions of Lemma 2.1, and the order of $G$ is 191. See Figure 9 for an embedding of $G$ in $\left(3.12^{2}\right)$. 


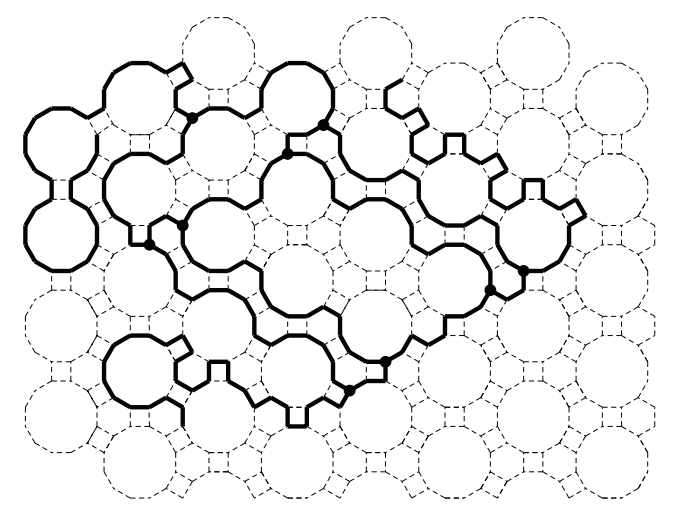

Figure 8: An embedding of $G$ in (4.6.12).

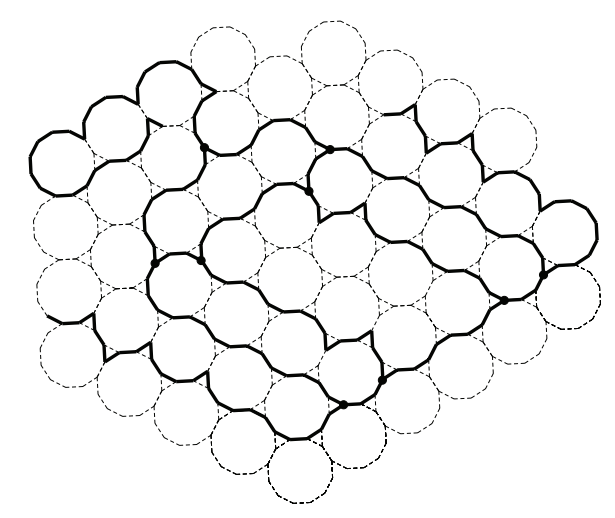

Figure 9: An embedding of $G$ in $\left(3.12^{2}\right)$. 


\section{Embeddings of graphs with connectivity 2}

In Figure 10 we show Zamfirescu's mentioned example $H^{\prime}$ from [16]. Let $H$ be a graph homeomorphic to $H^{\prime}$, depicted in Figure 11, where the letters indicate the numbers of consecutive vertices of degree 2 .

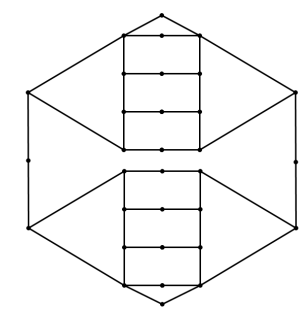

Figure 10: The graph $H^{\prime}$.

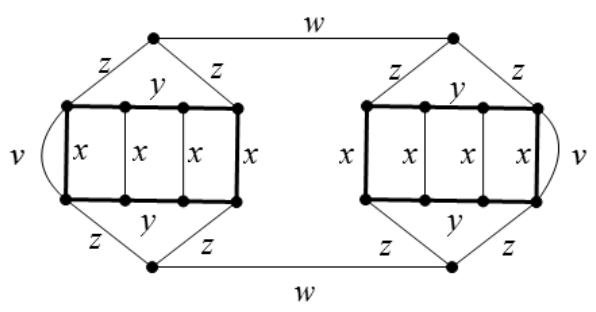

Figure 11: The graph $H$.

By the method used in the proof of Lemma 1 in [9], we have the following lemma, which is a modification of Lemma 2 in [9]. The proof is omitted again.

Lemma 3.1. Let $x \geq v$. The longest paths of $H$ have empty intersection if the following conditions are fulfilled.

(i) $v \geq y+2 z+1$,

(ii) $x+v=2 z+w+1$.

Theorem 3.2. There exists a 2-connected subgraph of $\left(3^{4} .6\right)$ of order 152 satisfying Gallai's property.

Proof. We use a particular case of Lemma 3.1. By setting $x=v=9, z=$ $2, y=0, w=13$, we get a graph $H$ of order 152 satisfying the conditions of 


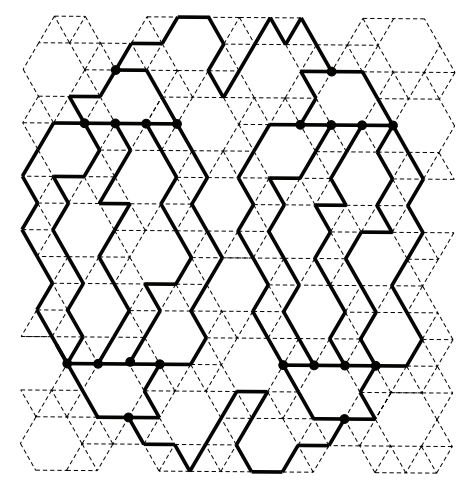

Figure 12: An embedding of $H$ in $\left(3^{4} .6\right)$.

Lemma 3.1. It can be embedded in the tiling graph $\left(3^{4} .6\right)$, as shown in Figure 12 .

Theorem 3.3. The Archimedean tiling graph $\left(3^{3} \cdot 4^{2}\right)$ contains a 2-connected subgraph of order 110 satisfying Gallai's property.

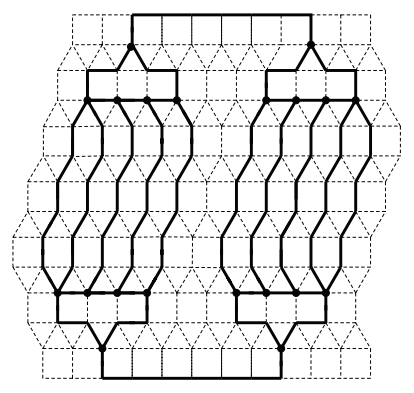

Figure 13: An embedding of $H$ in $\left(3^{3} \cdot 4^{2}\right)$.

Proof. If $x=v=6, z=2, y=0, w=7$, then the conditions in Lemma 3.1 are verified. The resulting graph $H$ of order 110 satisfies Gallai's property, and an embedding of $H$ in $\left(3^{3} .4^{2}\right)$ is shown in Figure 13.

Theorem 3.4. In the Archimedean tiling graph $\left(3^{2} .4 .3 .4\right)$ there is a 2-connected subgraph of of order 110 satisfying Gallai's property. 


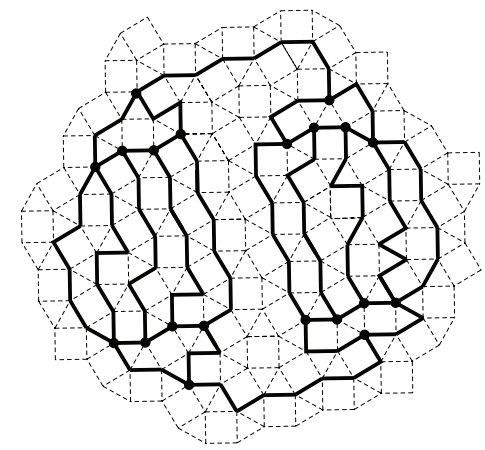

Figure 14: An embedding of $H$ in $\left(3^{2} .4 .3 .4\right)$.

Proof. Let $H$ be the graph described in Theorem 3.3. Figure 14 shows an embedding of $H$ in $\left(3^{2} .4 .3 .4\right)$.

Theorem 3.5. There exists a 2-connected subgraph of (3.6.3.6) of order 278 satisfying Gallai's property.

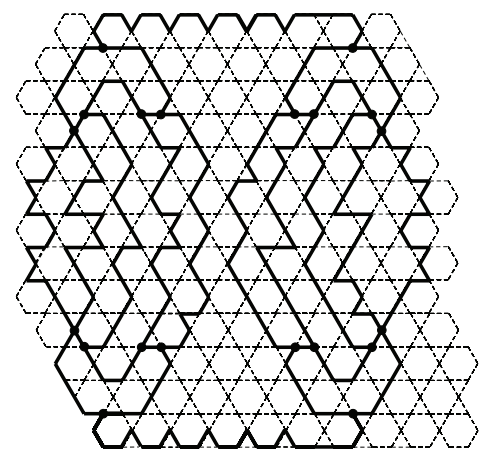

Figure 15: An embedding of $H$ in (3.6.3.6).

Proof. In Lemma 3.1 if we set $x=v=16, z=5, y=4, w=21$, then we have a graph $H$ of order 278, which satisfies the required property. Figure 15 shows an embedding of $H$ in (3.6.3.6).

Theorem 3.6. In the Archimedean tiling graph (3.4.6.4) there is a 2-connected subgraph of order 224 satisfying Gallai's property. 


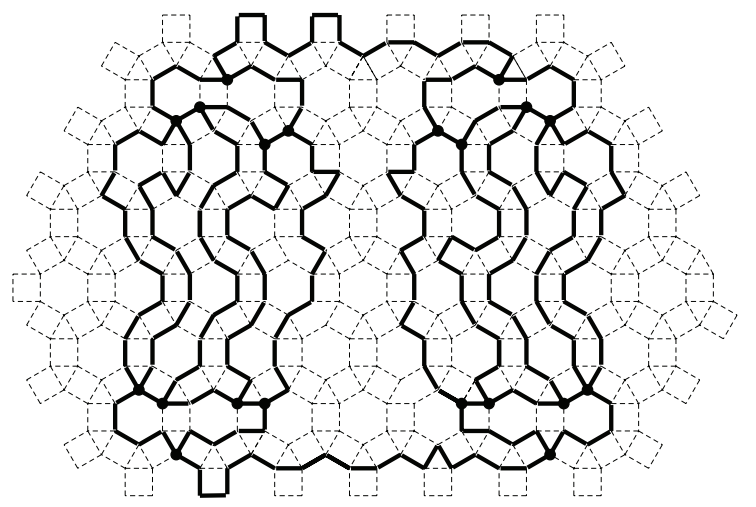

Figure 16: An embedding of $H$ in (3.4.6.4).

Proof. Put $x=v=13, z=4, y=2, w=17$ in Lemma 3.1. Then all the conditions are verified and we get a graph $H$ of order 224, satisfying Gallai's property. Now embed $H$ in (3.4.6.4), see Figure 16.

In order to find a graph with Gallai's property embeddable in the Archimedean tiling graphs $\left(4.8^{2}\right),(4.6 .12)$ and $\left(3.12^{2}\right)$, the graph $H$ and Lemma 3.1 don't work anymore since $H$ has vertices of degree 4 while all the Archimedean tiling graphs mentioned above are 3 -regular. Now consider the graph $K^{\prime}$ shown in Figure 17 (left side), and the graph $K$ which is homeomorphic to $K^{\prime}$, where $x, y, z, t, w$ and $m$ are numbers of vertices of degree 2 , as before.
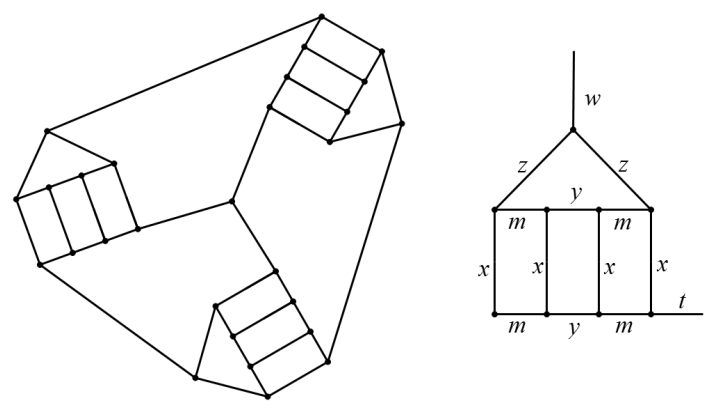

Figure 17: Graph $K^{\prime}$ and a subgraph of $K$.

Based on Lemma 3 in [9], we have the following lemma.

Lemma 3.7. Let $x \geq v$. The longest paths of $K$ have empty intersection if 
$y \geq 1, m \geq 1$ and $x=y+z-m \geq w=y+2 t-m+1$.

Theorem 3.8. There exists a 2-connected subgraph of $\left(4.8^{2}\right)$ of order 511 satisfying Gallai's property.

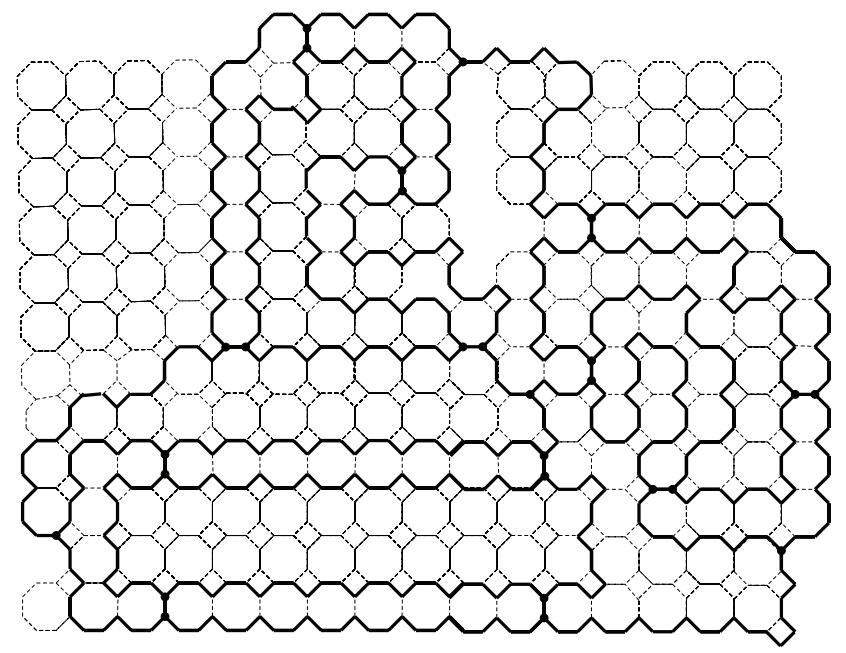

Figure 18: An embedding of $K$ in $\left(4.8^{2}\right)$.

Proof. It is clear that $K$ with $y=13, z=10, m=0, x=23, t=3, w=20$ satisfies the conditions of Lemma 3.7. It has order 511, and could be embedded in $\left(4.8^{2}\right)$, as shown in Figure 18 .

Theorem 3.9. In the Archimedean tiling graph (4.6.12) there is a 2-connected subgraph of order 541 satisfying Gallai's property.

Proof. In Lemma 3.7 let $y=13, z=11, m=2, x=22, t=5, w=22$. Then corresponding graph $K$ has order 541 and satisfies Gallai's property. An embedding of $K$ in (4.6.12) is shown in Figure 19.

Theorem 3.10. The Archimedean tiling graph $\left(3.12^{2}\right)$ contains a 2 -connected subgraph of order 499 satisfying Gallai's property.

Proof. It is easy to check that the conditions of Lemma 3.7 are satisfied if we take $y=11, z=11, x=y+z-m=20, t=5, m=2, w=y+2 t-m+1=20$. The resulting graph $K$ has 499 vertices. Figure 20 shows an embedding of $K$ in $\left(3.12^{2}\right)$. 


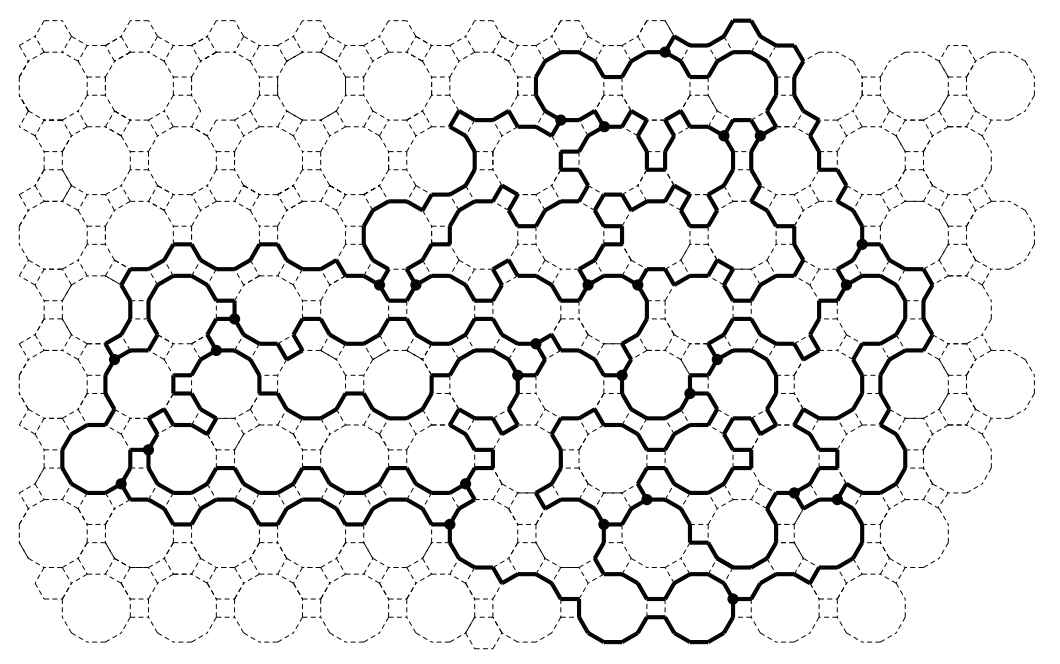

Figure 19: An embedding of $K$ in (4.6.12).

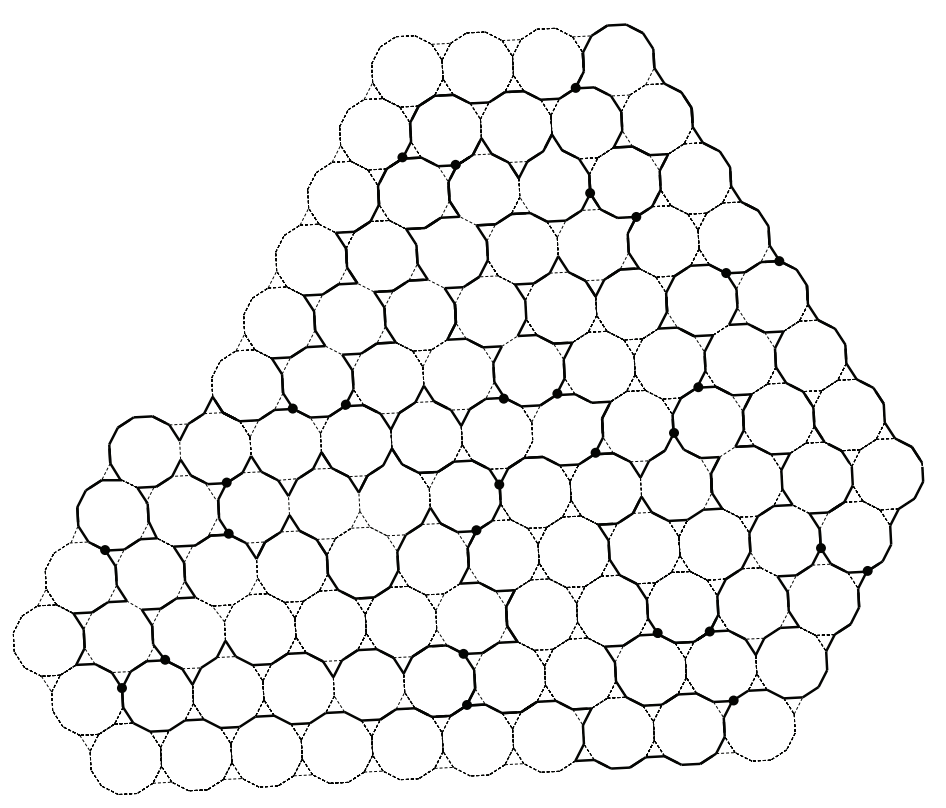

Figure 20: An embedding of $K$ in $\left(3.12^{2}\right)$. 
Acknowledgements. The second author gratefully acknowledges financial support by NSF of China (11471095), NSF of Hebei Province (A2013205189), and Program for Excellent Talents in University, Hebei Province (GCC2014043).

\section{References}

[1] Y. Bashir, T. Zamfirescu, Lattice graphs with Gallai's property, Bull. Math. Soc. Sci. Math. Roumanie 56 (2013) 65-71.

[2] T. Gallai, Problem 4, in: Theory of Graphs, Proc. Tihany 1966 (ed: P. Erdős and G. Katona), Academic Press, New York, 1968, 362.

[3] V. S. Gordon, Y. L. Orlovich and F. Werner, Hamiltonian properties of triangular grid graphs, Discrete Math. 308 (2008) 6166-6188.

[4] B. Grünbaum, Vertices missed by longest paths or circuits, J. Comb. Theory A 17 (1974) 31-38.

[5] Grünbaum, B., Shephard, G. C., Tilings and Patterns. New York: W. H. Freeman and Company, 1987.

[6] A. D. Jumani and T. Zamfirescu, On longest paths in triangular lattice graphs, Util. Math. 89 (2012) 269-273.

[7] S. Klavžar, M. Petkovšek, Graphs with non empty intersection of longest paths, Ars Combin. 29 (1990) 13-52.

[8] B. Menke, Ch. Zamfirescu and T. Zamfirescu, Intersections of longest cycles in grid graphs, J. Graph Theory 25 (1997) 37-52.

[9] F. Nadeem, A. Shabbir, and T. Zamfirescu, Planar lattice graphs with Gallais property, Graphs Combin. 29 (2013) 1523-1529.

[10] W. Schmitz, Über längste Wege und Kreise in Graphen, Rend. Sem. Mat. Univ. Padova 53 (1975) 97-103.

[11] Z. Skupień, Smallest sets of longest paths with empty intersection, Combin. Probab. Comput. 5 (1996), 429C436.

[12] W. T. Tutte, A theorem on planar graphs, Trans. Amer. Soc. 82 (1956) 99-116.

[13] H. Walther, Über die Nichtexistenz eines Knotenpunktes, durch den alle längsten Wege eines Graphen gehen, J. Comb. Theory 6 (1969) 1-6. 
[14] H. Walther and H.-J. Voss, Über Kreise in Graphen, VEB Deutscher Verlag der Wissenschaften, Berlin, 1974.

[15] T. Zamfirescu, A two-connected planar graph without concurrent longest paths, J. Combin. Theory B 13 (1972) 116-121.

[16] T. Zamfirescu, On longest paths and circuits in graphs, Math. Scand. 38 (1976) 211-239.

[17] T. Zamfirescu, Intersecting longest paths or cycles: a short survey, Analele Univ. Craiova, Ser. Mat. Inf. 28 (2001) 1-9.

Zhikui CHANG,

College of Mathematics and Information Science,

Hebei Normal University,

050024 Shijiazhuang, P.R. China.

and

Hebei Key Laboratory of Computational Mathematics and Applications, 050024 Shijiazhuang, P.R. China.

Email: changzhikui@163.com

Liping YUAN,

College of Mathematics and Information Science,

Hebei Normal University,

050024 Shijiazhuang, P.R. China.

and

Hebei Key Laboratory of Computational Mathematics and Applications, 050024 Shijiazhuang, P.R. China.

Email: lpyuan@hebtu.edu.cn 